\title{
BAC3 plays a central role in proteostasis in the heart
}

\author{
Wataru Mizushima and Junichi Sadoshima \\ Department of Cell Biology and Molecular Medicine, Cardiovascular Research Institute, Rutgers New Jersey Medical School, Newark, New Jersey, USA.
}

Proteinopathies are characterized by the accumulation of misfolded proteins, which ultimately interfere with normal cell function. While neurological diseases, such as Huntington disease and Alzheimer disease, are well-characterized proteinopathies, cardiac diseases have recently been associated with alterations in proteostasis. In this issue of the $J C I$, Fang and colleagues demonstrate that mice with cardiac-specific deficiency of the co-chaperone protein BCL2-associated athanogene 3 (BAC3) develop dilated cardiomyopathy that is associated with a destabilization of small HSPs as the result of a disrupted interaction between BAC3 and HSP70. Together, the results of this study suggest that strategies to upregulate BAC3 during cardiac dysfunction may be beneficial.

\section{Protein aggregates and disease}

Protein homeostasis (proteostasis) is essential for maintaining normal cell function (1, 2). Proper protein activity is achieved through rigorous coordination of synthesis, folding, and posttranslational modification, all of which are prone to malfunction in response to stress. For example, protein folding in the endoplasmic reticulum (ER) is easily disrupted in the presence of ER stress and oxidative stress. Disruption of proteostasis induces the accumulation of misfolded proteins, which then interfere with the normal function of cells through improper degradation, gain and/or loss of function, and aggregate formation. The adverse effects caused by altered proteostasis are collectively termed proteotoxicity, and pathological conditions resulting from proteotoxicity are collectively termed proteinopathies $(3,4)$. Proteostasis is particularly important for postmitotic cells, such as neurons and adult cardiomyocytes, that have negligible regenerative potential, because in these mature cells, proteotoxicity is not readily diluted through cell division. The concept of proteinopathy was initially used to explain the pathogenesis of neurodegenerative diseases, including Huntington disease and Alzheimer disease (5); however, several lines of evidence now suggest that proteotoxicity is also involved in the pathogenesis of many heart diseases, including cardiomyopathy and heart failure. Cells possess several protein quality control (PQC) mechanisms, comprising chaperones and protein degradation systems, including the ubiquitin-proteasome system (UPS) and autophagy-lysosome system, that help minimize proteotoxicity. Chaperones function as the first line of defense by either refolding misfolded proteins or by promoting the degradation of misfolded or damaged proteins that are not suitable for refolding via the UPS or autophagy. Accumulation of misfolded proteins occurs through dysregulation of PQC mechanisms, and the consequent proteotoxicity develops through many different mechanisms. In vivo animal models have proven quite useful in elucidating the molecular mechanisms of proteinopathy (6).

BCL2-associated athanogene 3 (BAG3) is a co-chaperone protein that regulates the ATPase activity of the HSP7O family and is predominantly expressed in striated muscles $(7,8)$. BAG3 has been studied extensively during the past decade, because, together with small HSPB8, it directs the HSP70 chaperone complex to other proteins involved in major cellular functions, thereby mediating key PQC mechanisms.

Related Article: p. 3189

Conflict of interest: The authors have declared that no conflict of interest exists

Reference information: / Clin Invest. 2017;127(8):2900-2903. https://doi.org/10.1172/JCI95839.

These mechanisms include aggresome formation through retrograde transport of misfolded proteins, which depends on the interaction of BAG3 with dynein motors and the microtubule organization center (9), and stimulation of autophagy, which depends on BAG3 interaction with HSPB8. Both retrograde transport and autophagy facilitate the sequestration and removal of irreversibly misfolded proteins. BAG3 is also involved in chaperone-assisted selective autophagy (CASA). CASA is a cargoselective form of autophagy that is mediated through the HSC70-BAG3-HSPB8 complex and allows ubiquitination of selected proteins recognized by HSC7O via the CHIP ubiquitin ligase and sequestration by autophagosomes through the LC3 adapter p62/ SQSTM in the presence of synaptopodin 2 or DNAJB6 (10). In muscles, BAG3 is particularly important for maintaining the structure and integrity of sarcomeres through its interaction with HSC7O and an actincapping sarcomeric protein (actin-capping protein $\beta 1$ ) (11). In mice, a homozygous null mutation of Bag3 causes a fulminant form of dilated cardiomyopathy (DCM) (12). In addition, a genome-wide association study (GWAS) identified several mutations in $B A G 3$, including an $\mathrm{E} 455 \mathrm{~K}$ mutation in the evolutionarily conserved BAG domain, that underlie a familial form of DCM (13). However, the molecular mechanism through which the loss of BAG3 function in cardiomyocytes leads to the development of DCM remains to be elucidated.

\section{Loss of BAG3 function alters $\mathrm{PQC}$ in murine DCM}

In this issue, Fang and colleagues used mice with cardiac-specific deletion of Bag3 (herein referred to as cKO mice) and mice with cardiac-specific expression of BAG3 ${ }^{\mathrm{E} 455 \mathrm{~K}}$ (herein referred to as cMUT mice) and demonstrated that loss of function of BAG3 induces the development of DCM by attenuating PQC over a specific subset of proteins involved in metabolic and contractile functions in cardiomyocytes (14). Downregulation of BAG3 or expression of BAG3 $3^{\mathrm{E} 455 \mathrm{~K}}$ disrupt- 
A WT

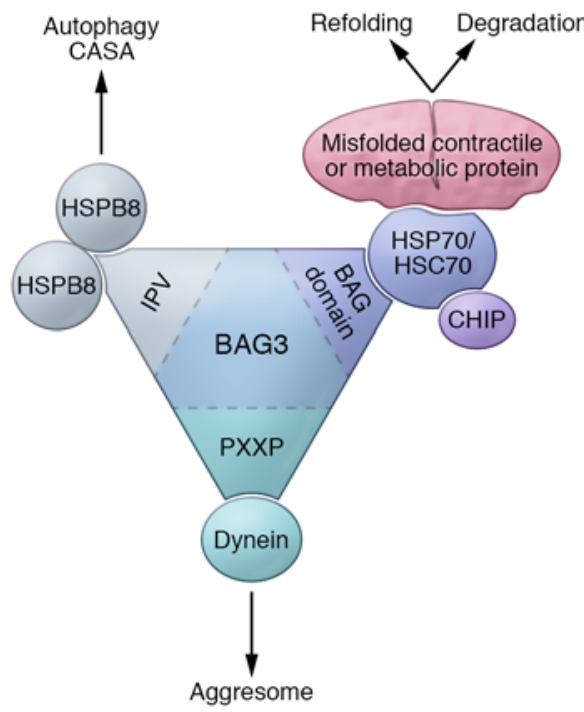

B E455K mutation

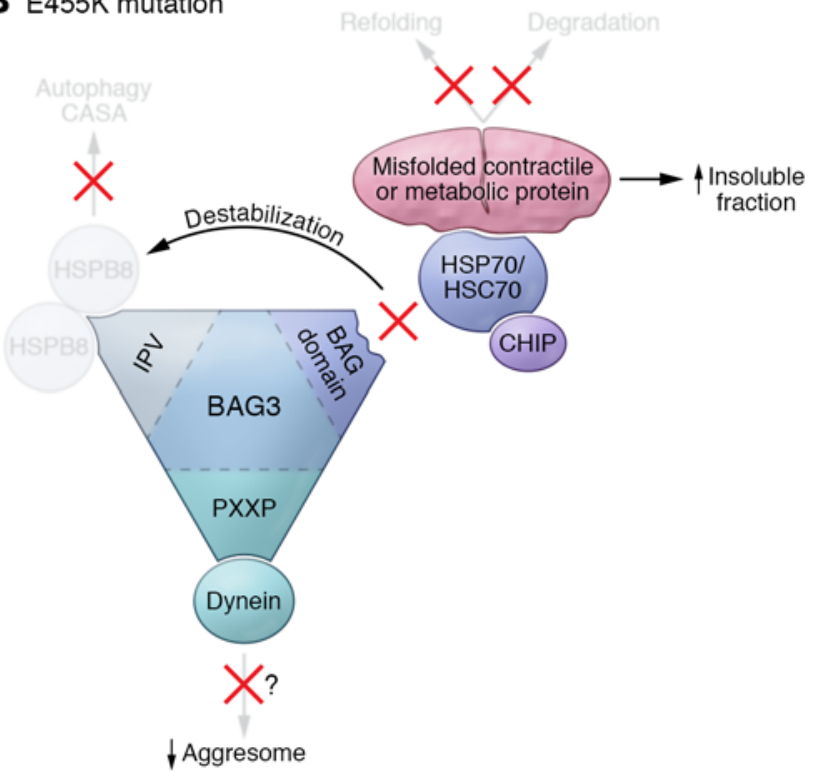

Figure 1. Putative functions of BAG3 in the heart and the underlying mechanisms of cardiomyopathy caused by loss of BAG3 function. BAC3 plays a critical role in the PQC system in the heart. WT BAG3 forms multi-chaperone complexes with HSP7O/HSC70 and sHSPs via its IPV motif and BAC3 domain. The BAG3-HSPB8-HSC70 complex binds to misfolded proteins and either promotes refolding or degradation of these misfolded proteins. Degradation is mediated primarily through general autophagy. The BAC3-HSPB8-HSC70 complex also maintains the structure and integrity of sarcomeres via CASA, a substrate-specific form of autophagy. In addition, BAC3 binds to dynein via the proline-rich motif (PXXP) and segregates misfolded proteins into aggresomes located in the perinuclear space through retrograde transport. The BAG3 ${ }^{\text {E455K }}$ mutation, which is located in the middle of the BAC domain, attenuates the interaction between BAC3 and HSP70/HSC70, leading to destabilization of sHSPs, including HSPB8. This disturbance of the chaperone complexes induces dysregulation of the refolding and degradation processes (autophagy and CASA), resulting in accumulation of BAC3 complex substrate proteins involved in metabolism and cardiac contraction in the detergent-insoluble fraction. Interestingly, aggresomes are not obvious in the BAC3 loss-of-function mouse model, possibly because of the defect in retrograde transport. Despite the absence of large protein aggregates, BAC3 loss-of-function mice develop DCM.

ed the interaction between BAG3 and HSP7O and consequently downregulated several small HSPs (sHSPs), including HSPB5, HSPB6, and HSPB8, through protein destabilization. As a result, both protein refolding and degradation of selected HSP70-BAG3-HSPB8 complex-interacting proteins, many of which are involved in metabolism and cardiac contraction, were suppressed, resulting in accumulation of these proteins in the detergent-insoluble fraction (Figure 1).

Interestingly, large protein aggregates were not observed in cKO or cMUT mice, despite a drastic increase in the amount of proteins in the detergent-insoluble fraction. As BAG3 and HSPB8 are critical regulators of dynein-mediated retrograde transport (15), aggresome formation may be actively suppressed in these loss-offunction mouse models. As mentioned by Fang et al., the lack of aggresomes in BAG3-deficient mice strongly suggests that small and invisible protein aggregates, rather than large protein aggregates, are sufficient for the development of cardiac dysfunction. This proposed contribution of small aggregates to cardiac dysfunction in cKO and cMUT mice is similar to a study that demonstrated that the presence of soluble preamyloid oligomers (PAOs) is sufficient to induce cardiomyocyte dysfunction in desmin cardiomyopathy mouse models (16). It is possible that aggresome formation through BAG3-mediated retrograde transport may even be an adaptive process to protect cardiomyocytes from the toxicity caused by small protein aggregates.

\section{Remaining questions and future directions}

Although the study by Fang et al. clearly suggests that proper coordination of the actions of HSP70, BAG3, and HSPB8 is essential for the maintenance of PQC in the adult heart at baseline, several important issues remain to be addressed. For example, it remains unclear whether the selected proteins that accumulate in the detergent-insoluble fraction act as toxic molecules on their own. In a zebrafish model of myofibrillar myopa- thy, overexpression of BAG3 ${ }^{\mathrm{P} 209 \mathrm{~L}}$ induces the formation of myofibrillar aggregates that sequester functional WT BAG3, thereby acting in a toxic gain-of-function manner (17). More extensive ultrastructural and biochemical analyses of the proteotoxicity associated with the different BAG3 mutants and of the presence of preamyloid oligomers would be helpful to address this issue. Alternatively, the depletion of some proteins in the soluble fraction may be more important and directly cause the functional deficit in the BAG3 loss-of-function mouse models. Fang et al. show that a limited number of proteins involved in muscle contraction, actin filament capping, protein folding, mitochondrial organization, and cell junction assembly are shifted to the insoluble fraction as a result of BAG3 deficiency. Interestingly, there does not appear to be any compensation for the decreased protein level within the soluble faction. Although BAG3 is capable of compensating for the loss of proteins degraded through autophagy by activat- 
ing mTOR or YAP/TAZ (18), these compensatory mechanisms are apparently missing in the BAG3 loss-of-function mouse models. Future studies should test whether restoring the level of selected proteins in the soluble fraction can rescue cardiac dysfunction in the cKO and cMUT mouse models.

BAG3 has pleiotropic functions in cardiomyocytes due to the presence of multiple functional domains, including the WW domain, the IPV (Ile-Pro-Val) motif, the proline-rich motif, and the BAG domain, that interact with distinct sets of proteins. It is not clear how or which of the different BAG3 domains contribute to the overall cardiac phenotype observed in the BAG3 loss-of-function mouse models, especially because these domains are involved in protein refolding, aggresome formation, protection against apoptosis, and stimulation of both macroautophagy and CASA. For example, BAG3 inhibits apoptosis by interacting with the antiapoptotic protein BCL-2 through the BAG domain (19). Additional experiments are needed to determine whether cardiac apoptosis contributes to the cardiac systolic dysfunction and heart failure found in BAG3-deficient mice. BAG3-mediated activation of general autophagy and CASA are expected to degrade different sets of cargo targets. In addition, a loss of BAG3 function is often compensated for by BAG1, which activates degradation through the UPS (20). It would be interesting to elucidate how the loss of general autophagy and CASA contributes to and how the compensatory activation of the UPS affects overall protein degradation in the BAG3 loss-of-function mice.

Finally, BAG3 is downregulated in response to heart failure in both mice and humans, as well as in a subset of patients with idiopathic DCM $(14,21)$. Although heart failure is often accompanied by attenuation of the PQC mechanisms and increased protein misfolding, it remains to be determined whether downregulation of BAG3 substantially contributes to decreased PQC during heart failure. While BAG3 is a stress-responsive protein, its expression is reduced in epinephrine stress-induced Takotsubo cardiomyopathy patients with a polymorphism in the 3 '-UTR of BAG3 (22). BAG3 coding variants at position 81 , either methionine or isoleucine, have been shown to alter BAG3 function. Specifically, BAG3 $3^{\mathrm{M} 81}$ has been shown to have improved binding to HSPB8 and to increase autophagy in limb muscles in response to ischemia compared with BAG3 ${ }^{\mathrm{I} 11}(23)$. These results are consistent with the notion that the PQC mechanisms controlled by endogenous BAG3 are involved in protection against myocardial stress. In fact, upregulation of BAG3 improves cardiac function in a mouse model of chronic myocardial infarction and reduces cardiomyocyte death in response to ischemia and reperfusion (8). Thus, interventions to upregulate the level of endogenous BAG3 should be further considered for treating diseases associated with cardiac stress. Transcription of $B A G 3$ is regulated by $\mathrm{NF}-\kappa \mathrm{B}$ and miR-371a-5p and in response to physical exercise (8). Interestingly, although both exercise and overexpression of ATG7 attenuate intracellular protein aggregates by inducing autophagy in desmin-related cardiomyopathy, exercise and ATG7 overexpression have the additive effects of improving cardiac function in another model of proteinopathy (24). Thus, combining multiple methods to enhance autophagy may allow for better control of cardiomyopathy caused by loss of function of BAG3.

\section{Acknowledgments}

The authors thank Daniela Zablocki (Rutgers New Jersey Medical School, Newark, NJ, USA) for critical reading of the manuscript. This work was supported in part by US Public Health Service grants HL67724, HL91469, HL102738, HL112330, and AG23039 (to JS) and by the Leducq Foundation Transatlantic Network of Excellence (to JS).

Address correspondence to: Junichi Sadoshima, Rutgers New Jersey Medical School, Department of Cell Biology and Molecular Medicine, 185 South Orange Ave, MSB G-609, Newark, New Jersey 07103, USA. Phone: 973.972.8920; Email: sadoshju@njms.rutgers.edu.

1. McLendon PM, Robbins J. Proteotoxicity and cardiac dysfunction. Circ Res. 2015;116(11):1863-1882.

2. Wang ZV, Hill JA. Protein quality control and metabolism: bidirectional control in the heart. Cell Metab. 2015;21(2):215-226.

3. Wang X, Robbins J. Proteasomal and lysosomal protein degradation and heart disease. JMol Cell Cardiol. 2014;71:16-24.

4. Willis MS, Patterson C. Proteotoxicity and cardiac dysfunction - Alzheimer's disease of the heart? NEngl J Med. 2013;368(5):455-464.

5. Ross CA, Poirier MA. Protein aggregation and neurodegenerative disease. Nat Med. 2004;10(Suppl):S10-S17.

6. Sanbe A, et al. Desmin-related cardiomyopathy in transgenic mice: a cardiac amyloidosis. Proc Natl Acad Sci US A. 2004;101(27):10132-10136.

7. Behl C. Breaking BAG: The co-chaperone BAG3 in health and disease. Trends Pharmacol Sci. 2016;37(8):672-688.

8. Knezevic T, et al. BAG3: a new player in the heart failure paradigm. Heart Fail Rev. 2015;20(4):423-434.

9. Gamerdinger M, Kaya AM, Wolfrum U, Clement AM, Behl C. BAG3 mediates chaperone-based aggresome-targeting and selective autophagy of misfolded proteins. EMBO Rep. 2011;12(2):149-156.

10. Ulbricht A, et al. Cellular mechanotransduction relies on tension-induced and chaperone-assisted autophagy. Curr Biol. 2013;23(5):430-435.

11. Hishiya A, Kitazawa T, Takayama S. BAG3 and Hsc7O interact with actin capping protein CapZ to maintain myofibrillar integrity under mechanical stress. Circ Res. 2010;107(10):1220-1231.

12. Homma S, Iwasaki M, Shelton GD, Engvall E, Reed JC, Takayama S. BAG3 deficiency results in fulminant myopathy and early lethality. Am J Pathol. 2006;169(3):761-773.

13. Villard E, et al. A genome-wide association study identifies two loci associated with heart failure due to dilated cardiomyopathy. Eur Heart J. 2011;32(9):1065-1076.

14. Fang $X$, et al. Loss-of-function mutations in co-chaperone BAG3 destabilize small HSPs and cause cardiomyopathy. J Clin Invest. 2017;127(8):3189-3200.

15. Zhang X, Qian SB. Chaperone-mediated hierarchical control in targeting misfolded proteins to aggresomes. Mol Biol Cell. 2011;22(18):3277-3288.

16. Pattison JS, Sanbe A, Maloyan A, Osinska H, Klevitsky R, Robbins J. Cardiomyocyte expression of a polyglutamine preamyloid oligomer causes heart failure. Circulation. 2008;117(21):2743-2751.

17. Ruparelia AA, Oorschot V, Vaz R, Ramm G, Bryson-Richardson RJ. Zebrafish models of BAG3 myofibrillar myopathy suggest a toxic gain of function leading to BAG3 insufficiency. Acta Neuropathol. 2014;128(6):821-833.

18. Kathage B, et al. The cochaperone BAG3 coordinates protein synthesis and autophagy under mechanical strain through spatial regulation of mTORC1. Biochim Biophys Acta. 2017;1864(1):62-75.

19. Takayama S, Xie Z, Reed JC. An evolutionarily conserved family of $\mathrm{Hsp} 70 / \mathrm{Hsc70}$ molecular chaperone regulators. J Biol Chem. 1999;274(2):781-786.

20. Gamerdinger M, Hajieva P, Kaya AM, Wolfrum U, Hartl FU, Behl C. Protein quality control 
during aging involves recruitment of the macroautophagy pathway by BAG3. EMBO J. 2009;28(7):889-901.

21. Feldman AM, et al. Decreased levels of BAG3 in a family with a rare variant and in idiopathic dilated cardiomyopathy. JCell Physiol. 2014;229(11):1697-1702
22. d'Avenia M, et al. A novel miR-371a-5p-mediated pathway, leading to BAG3 upregulation in cardiomyocytes in response to epinephrine, is lost in Takotsubo cardiomyopathy. Cell Death Dis. 2015;6:e1948.

23. McClung JM, et al. A BAG3 coding variant in mice determines susceptibility to ischemic limb muscle myopathy by directing autophagy [published online ahead of print April 25, 2017]. Circulation. https://doi.org/10.1161/ CIRCULATIONAHA.116.024873.

24. Bhuiyan MS, et al. Enhanced autophagy ameliorates cardiac proteinopathy. JClin Invest. 2013;123(12):5284-5297. 